\title{
Wavelet \& Other Thresholding Technique in Digital Image Processing
}

\author{
Deepti Rai ${ }^{1}$, Vaishali Suryavanshi ${ }^{2}$ \\ ${ }^{1}$ Professor Electronics \& Communication Engineering, Department, Alpine Institute of Technology (AIT), Ujjain (M.P) \\ ${ }^{2}$ PG Student, Department of Electronics and Communication Engineering, (AIT), Ujjain (M.P)
}

\begin{abstract}
The past decade has witnessed the development of wavelet analysis, a new tool that emerged from mathematics and was quickly adopted by diverse fields of science and engineering. In the brief period since its creation in 1987-88, it has reached a certain level of maturity as a well-defined mathematical discipline, with its own conferences, journals, research monographs, and textbooks proliferating at a rapid rate. Wavelet analysis has begun to play a serious role in a broad range of applications, including signal processing, data and image compression, solution of partial differential equations, modelling multiscale phenomena, and statistics. There seem to be no limits to the subjects where it may have utility. Our aim is to explore some additional topics that extend the basic ideas of wavelet analysis.
\end{abstract}

Keywords: discrete wavelet transform, image processing, noise removal, median filter, PSNR

\section{Introduction to wavelet packet transform}

A wavelet packet transform is a simple generalization of a wavelet transform. In this section I discussed the definition of wavelet transforms, and in the next section examine some examples illustrating their applications.

All wavelet packet transforms are calculated in a similar way. Therefore we shall concentrate initially on the Haar wavelet packet transform, which is the easiest to describe. The Haar wavelet packet transform is usually referred to as the Walsh transform. [2]The Discrete Fourier Transform (DFT) may be thought of in general terms as a matrix multiplication in which the original vector ${ }^{x_{k}}$ is decomposed into a series of coefficients $X_{n}$. Both k and $\mathrm{n}$ are integers which range over the same value $\mathrm{N}$.

$$
\left(\begin{array}{c}
x_{0} \\
\cdot \\
\cdot \\
x_{N-1}
\end{array}\right)=\left(\begin{array}{cccc}
\cdot & \cdot & \cdot & \cdot \\
\cdot & W_{k n} & \cdot & \cdot \\
\cdot & \cdot & \cdot & \cdot \\
\cdot & \cdot & \cdot & \cdot
\end{array}\right)\left(\begin{array}{c}
X_{0} \\
\cdot \\
X_{N-1}
\end{array}\right)
$$

In the above we may derive the transformed coefficients $X_{n}$ by inverting the matrix. The form of $W_{k n}$ has many possibilities but physically we would like the option of forward and backward transforms ie., an inverse ought to exist. The Discrete Wavelet Transform (DWT) generates a matrix $W_{k n}$ which is now widely used for image compression instead of the FT since it is able to localise preserve photographic detail such that many of the coefficients may be ignored (tantamount to filtering) and yet the reconstruction remains effective. DWT's are particularly effective in analysing waveforms which have spikes or pulses buried in noise. The noise may be more effectively removed than with FT filtering and the shape of the pulses preserved. Conservation of Energy similar to a Parseval theorem would also be nice.

\section{Haar Wavelet Transform}

Suppose for simplicity we assume an input vector $x_{k}$ with $0<k<7$. This is readily decomposed into an obvious basis set as shown below

$$
\left(x_{k}\right)=x_{0}\left(\begin{array}{l}
1 \\
0 \\
0 \\
0 \\
0 \\
0 \\
0 \\
0
\end{array}\right)+x_{1}\left(\begin{array}{l}
0 \\
1 \\
0 \\
0 \\
0 \\
0 \\
0
\end{array}\right)+x_{2}\left(\begin{array}{l}
0 \\
0 \\
0 \\
0 \\
0 \\
0 \\
0
\end{array}\right) \ldots+x_{7}\left(\begin{array}{l}
0 \\
0 \\
0 \\
0 \\
0 \\
1
\end{array}\right)
$$

In 1910 Haar proposed the following decomposition.

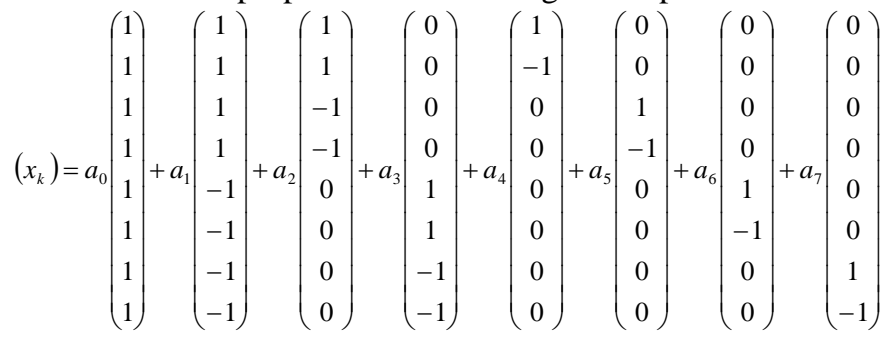

or $x_{n}=H_{n k} a_{k}$ with the columns of $\mathrm{H}$ being simply the

above basis vectors and the $a_{k}$ obtained by matrix inversion of H.These basis vectors have characteristic "shapes" when 


\section{International Journal of Science and Research (IJSR) \\ ISSN (Online): 2319-7064 \\ Index Copernicus Value (2015): 78.96 | Impact Factor (2015): 6.391}

drawn on their side as shown in the figure on the next page and it is these shapes which show the essential features of what DWT decomposition does.

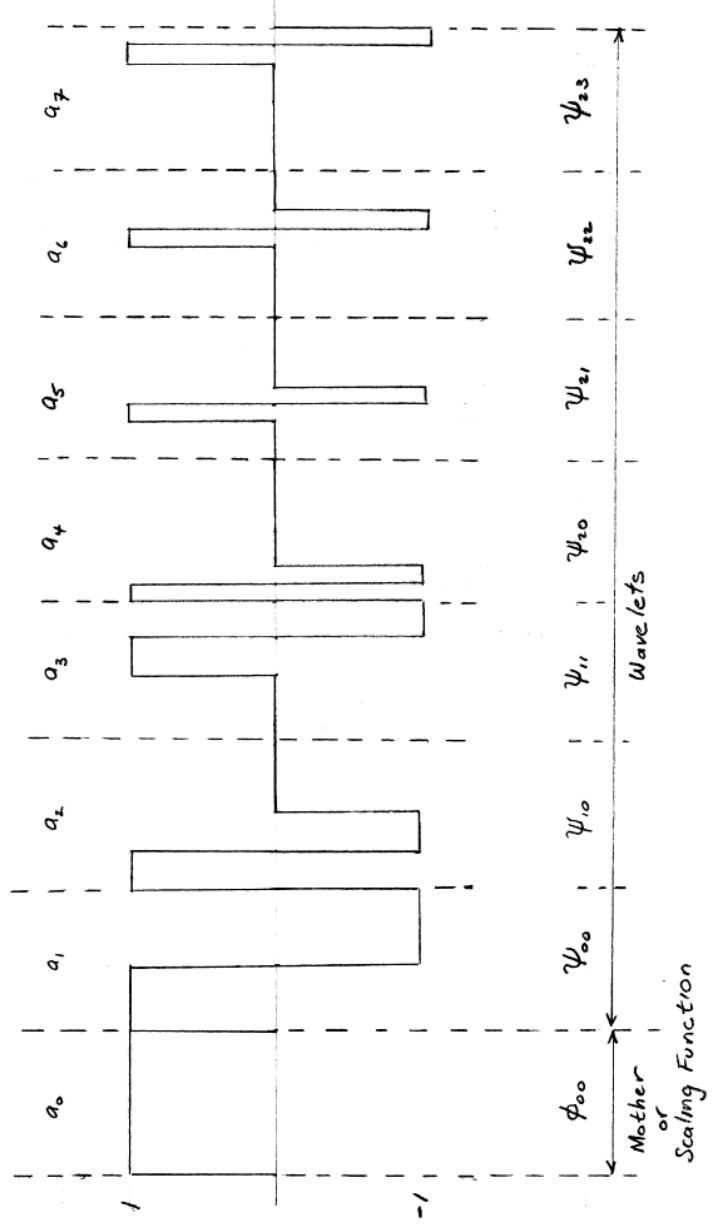

Figure 1: Wavelet decomposition chart

Notice:

1) A mother or scaling function at the start with a non-zero average. This will normally be normalised to 1 .

2) Wavelet functions with zero average which are both compressed and translated. It is this compression and translation which finds peaks or pulses well.

3) The wavelet functions are orthogonal. You can see this directly by multiplying any two together.

4) The wavelet functions have compact support which means they are all localised. This is unlike the FT in which the basis functions $\exp (-2 \pi n k / N)$ are continuous.

The Haar orthogonal system begins with $\Phi$ (t), the characteristic function of the unit interval

$$
\Phi(\mathrm{t})=\mathrm{x}_{[0,1)}(\mathrm{t})
$$

It is clear that $\Phi(\mathrm{t})$ and $\Phi(\mathrm{t}-\mathrm{n}), \mathrm{n} \neq 0, \mathrm{n} \in \mathrm{Z}$ are orthogonal since their product is zero. It is also clear that $\{\phi(\mathrm{t}-\mathrm{n})\}$ is not a complete orthogonal system in $\mathrm{L}^{2}(\mathrm{R})$ since its closed linear span $V_{o}$ consists of 2 piecewise constant functions with possible jumps only at the integers. The characteristic function of $(0,1 / 2)$, for example, with a jump at $1 / 2$, can not have a convergent expansion.

In order to include more functions we consider the dilated version of $\phi(\mathrm{t})$ as well, $\phi\left(2^{\mathrm{m}} \mathrm{t}\right)$ where $\mathrm{m} \in \mathrm{Z}$. Then by a change of variable we see that $\left\{2^{\mathrm{m} / 2} \phi\left(2^{\mathrm{m}} \mathrm{t}-\mathrm{n}\right)\right\}$ is an orthonormal system. $V_{m}$ will denote its closed linear span. Since any function in $\mathrm{L}^{2}(\mathrm{R})$ may be approximated by a piecewise constant function $\mathrm{f}_{\mathrm{m}}$ with jumps at binary rationals, it follows that is dense in $\mathrm{L}^{2}(\mathrm{R})$. Thus the system $\left\{\phi_{\mathrm{mn}}\right\}$ where

$$
\phi_{\mathrm{mn}}(\mathrm{t})=2^{\mathrm{m} / 2} \Phi\left(2^{\mathrm{m}} \mathrm{t}-\mathrm{n}\right)
$$

is complete in $\mathrm{L}^{2}(\mathrm{R})$, but, since $\phi(\mathrm{t})$ and $\phi(2 \mathrm{t})$ are not orthogonal, it is not an orthogonal system. We must modify it somehow to convert it into an orthogonal system.

Fortunately the cure is simple; we let $\Psi(\mathrm{t})=\phi(2 \mathrm{t})-\phi(2 \mathrm{t}-$ 1). Then everything works; $\{\psi(\mathrm{t}-\mathrm{n})\}$ is orthonormal system, and $\psi(2 \mathrm{t}-\mathrm{k})$ and $\psi(\mathrm{t}-\mathrm{n})$ are orthogonal for all $\mathrm{k}$ and $\mathrm{n}$. This enables us to deduce that $\left\{\psi_{\mathrm{mn}}\right\}_{\mathrm{m}, \mathrm{n} \in \mathrm{Z}}$, where

$$
\psi_{\mathrm{mn}}(\mathrm{t})=2^{\mathrm{m} / 2} \psi\left(2^{\mathrm{m}} \mathrm{t}-\mathrm{n}\right) \text {, }
$$

is a complete orthonormal system in $\mathrm{L}^{2}(\mathrm{R})$. this is the Haar system; the expansion of $\mathrm{f} \in \mathrm{L}^{2}(\mathrm{R})$ is

$$
f(t)=\sum_{m=-\infty}^{\infty} \sum_{n=-\infty}^{\infty}\left\langle f, \psi_{m n}\right\rangle \psi_{m n}(t)
$$

\section{Recent Works Done on Wavelet Transforms and Image Processing}

In 2014 Arpita and etal and others in their research paper titled "impact of wavelet transform and median filtering on removal of salt and pepper noise in digital images" explained Image acquisition is a common task in every image processing operation. Noise is entered during image acquisition from its source and once entered it degrades the image and is difficult to remove. In order to achieve the noise cancellation in an image, non-linear filter works better than linear. This paper presents the joint scheme of Wavelet Transform using iterative noise density and Median Filtering to remove Salt and Pepper Noise in Digital Images. The first part of the paper derives the wavelet coefficients with slight increase in noise density and in second part these coefficients are further modified by median filter. The algorithm shows the remarkable improvement over Gaussian noise model and removes most of the noisy part from the image and maintains the visual quality. The level of wavelet decomposition is restricted to three. The renowned indexes Peak Signal to Noise Ratio (PSNR) and Root Mean Square Error (RMSE) demonstrate marked improvement of image denoising over Gaussian method.

In 2014 Sezal Khera and others in their research paper titled "survey on medical image de noising using various filters and wavelet transform" explained that There is a problem of high 


\section{International Journal of Science and Research (IJSR) \\ ISSN (Online): 2319-7064 \\ Index Copernicus Value (2015): 78.96 | Impact Factor (2015): 6.391}

level components of noises in the Medical Images. The different medical images are Magnetic Resonance Imaging (MRI), X-ray, Computed Tomography and Ultrasound. There are many noise reduction techniques that have been developed for removing noise. The idea behind these techniques is to get better results in terms of quality and in removal of different noises. This paper gives the review of various de noising techniques.

In 2014 Seema and others in their research paper titled "wavelet based technique for removal of multiple noises simultaneously" presented that Denoising is important preprocessing tasks for various image processing. Image noise is the random variation of brightness and color information in images produced by the scanner and digital camera. This paper presents a novel approach for simultaneously removing the speckle and Salt-n-pepper noise from a single image by using the median filter. This paper proposes an adaptive, data-driven threshold for image denoising via wavelet soft thresholding. The threshold is derived in a Bayesian framework and also using the MAD (mean absolute difference) value of fast multidirectional filter bank which improves the radial frequency resolution of the image by addition decomposition in the high frequency band. Denoising performance of median filter will be compared with discrete wavelet transform and wiener filter. The performance of median filter using parameter metrics PSNR (Peak Signal to Noise Ratio) and coefficient of correlation (Coc) is also analyzed.

In 2013 Himanshu and others in their research paper titled "Image denoising by hybrid average gaussian filter for different noises" presented the study of four types of noises such as Gaussian, Salt and Pepper, Poisson, and Speckle noise. Image denoising is not only significant on the contrary problem of image processing which is helpful in the fields of remotely sensed scene interpretation, Biomedical imaging techniques, Gathering image's lost information, image retrieval, mining of image etc, and an essential preprocessing technique to preserve the clarity of the naturally corrupted image which may be affected by the various types of noises. This paper reviews on the existing various filters having various variance of noises. Further, it analyses, examines and compares various filters with the proposed filter. The experimental results shown to be precised in terms of SNR and variance in noise. The results consider the quantitative measures of comparing the denoised images as output of various filters and the hybrid filter with the help of Root Mean Square Errors (RMSE) and Peak-Signal to Noise Ratio (PSNR).

In 2010 Pawan and others in their research paper titled "1mage de-noising by various filters for different noise" presented that Image processing is basically the use of computer algorithms to perform image processing on digital images. Digital image processing is a part of digital signal processing. Digital image processing has many significant advantages over analog image processing. Image processing allows a much wider range of algorithms to be applied to the input data and can avoid problems such as the build-up of noise and signal distortion during processing of images. Wavelet transforms have become a very powerful tool for de-noising an image. One of the most popular methods is wiener filter. In this work four types of noise (Gaussian noise , Salt \& Pepper noise, Speckle noise and Poisson noise) is used and image de-noising performed for different noise by Mean filter, Median filter and Wiener filter . Further results have been compared for all noises.

\section{Research Methodology and Problem Statement}

In all the literature that we have looked so far are either using one technique for noise removal, or they are using multiple noises to study a single technique. Also it has been found that work on de noising an image has been done through laplasian, wavlet and other traditional methods but the methods that we have seen in section II \& III are currently not used that much for image de noising.

Keeping this in mind we propose a new approach that would include the use of following technique :-

- Haar transform

- Walsch transform

- Maxican hat wavelet

We will study de noising based on the above methods on the following types of noises :-

- Gaussian,

- Salt and Pepper,

- Poisson, and

- Speckle noise

Then we will perform a comparative analysis of the above approaches for de noising a particular image.

\section{Conclusion}

Wavelet-based image compression method using in JPEG2000 is the new standard for still image compression. It provides a new framework and an integrated toolbox to better address increasing needs for compression. It also provides a wide range of functionalities for still image applications. Lossless and lossy coding, embedded lossy to lossless, progressive by resolution and quality, high compression efficiency, error resilience and lossless color transformations are some of its characteristics. Comparative results have shown that JPEG2000 is indeed superior to existing still image compression standards. Work is still needed in optimizing its implementation performance. Denoising is the concept of removing the noises by using the various types of filters and techniques. For this purpose, a new method based on discrete wavelet transform using the bayesshrink method was proposed and the results were compared with already established median and wiener filter. By using proposed technique, two noises, namely Salt \&Pepper and Gaussian 


\section{International Journal of Science and Research (IJSR) \\ ISSN (Online): 2319-7064 \\ Index Copernicus Value (2015): 78.96 | Impact Factor (2015): 6.391}

noise, were reduced simultaneously from a single image were removed successfully and results were found to be better than wiener and median filters due to better PSNR ratio and Coc value. Results revealed that the proposed method was very efficiently able to remove noise from ultrasound gray scale images then others images so this proposed technique perform better result on ultrasound images. Wavelet based denoising algorithms uses soft and hard thresholding to provide smoothness and better edge preservation.

\section{References}

[1] E.J. Leavline and S. Sutha, "Gaussian Noise Removal in Gray Scale Images Using fast Multiscale Directional Filter Bank", IEEE International Conference in Information Technology, 2011, pp. 884889.

[2] P. Patidar, M. Gupta, S. Srivastava and A.K. Nagawat, "Image De-noising by various Filters for Different noise", International Journal of Computer Applications, vol. 9, No.4, 2011, pp.45-50.

[3] G. Ilango and R. Marudhachalam, "New Hybrid filtering techniques for removal of Gaussian noise from medical images", ARPN Journal of Engineering and Applied Science, Vol. 6, No.2, 2011, pp. 8-12.

[4] J.K. Mandal and A. Sarkar, "A modified weighted based filter for removal of random impulse noise", IEEE Emerging Applications of Information Technology, 2011, pp. 173-176.

[5] K. Karthikeyan and C. Chandrasekar, "Speckle Noise Reduction of Medical Ultrasound Images using Bayesshrink Wavelet Threshold", International Journal of Computer Applications, Vol. 22, No. 9, 2011, pp. 814.

[6] S.Arivazhagan, S. Deivalakshmi and K. Kannan, "Performance Analysis of image denoising system for different levels of wavelet decomposition", International Journal of Imaging Science and Engineering, Vol. 1, No. 3, 2007, pp. 104-107.

[7] D. Maheswari and V. Radha, "Noise Removal In Compound Image Using Median Filter", International Journal on Computer Science and Engineering, Vol. 2, No. 4, 2010, pp.1359-1362.

[8] I Shanthi, "Speckle Noise Suppression of SAR Colour Image using Hybrid Mean Median Filter ", International Journal of Computer Applications(09758887) Volume -31 No. -9 October 2011

[9] Amandeep Kaur, Karamjeet Singh , "Speckle noise reduction by using Wavelets ", NCCI 2010 -National Conference on Computational Instrumentation CSIO Chandigarh, INDIA, 19-20 March 2010.

[10] Pavan Patidar, Manoj Gupta, Sumit, Ashok, "Image Denoising By Various Filters For Different Noises", International Journal of Computer Applications (0975 8887)Volume 9-No.4, November 2010.

[11] Reena Thakur, Shweta, Rishu, Supriya, "Selection of Wavelet from Wavelet families to facilitate the evolution of Color Image Denoising", International Journal of Advance Research, Vol 1, Issue 2, May 2013, ISSN 23209194.

[12] Reena Thakur, Arun Yadav, "Hybrid Segmentation Approach and Preprocessing of Color Image based on Haar Wavelet Transform", International Journal of Computer Applications 46(16):1-5, May 2012, Published by Foundation of Computer Science, New York, USA.

Volume 6 Issue 7, July 2017 www.ijsr.net 